\title{
A linear model for amplitude modulation of Langmuir waves in weak electron-beam plasma interaction
}

\author{
K. Baumgärtel \\ Leibniz-Institut für Astrophysik Potsdam, An der Sternwarte 16, 14482 Potsdam, Germany \\ Correspondence to: K. Baumgärtel (uk.baumgaertel@t-online.de)
}

Received: 17 August 2012 - Revised: 1 November 2012 - Accepted: 5 December 2012 - Published: 4 January 2013

\begin{abstract}
A simple linear approach to the phenomenon of amplitude modulation of Langmuir waves in weak beam plasma interaction is presented. During the short growth phase of the instability and within the longer period after saturation, the waves are described by their linear kinetic dispersion properties. The amplitude modulation appears as result of the beating of waves with different wavelengths and amplitudes that have grown from noise in the initial phase. The Langmuir wave fields are calculated via FFT (fast Fourier transform) technique. The resulting waveforms in temporal representation are quite similar to those observed by spacecraft.
\end{abstract}

Keywords. Space plasma physics (Waves and instabilities)

\section{Introduction}

Amplitude modulation of electron-beam-driven Langmuir waves has been observed under different space plasma conditions by many spacecraft during the last three decades. Among them are Voyager (Gurnett et al., 1981); Galileo (Gurnett et al., 1993; Hospodarsky et al., 1994); Wind (Kel$\log$ et al., 1996); Geotail (Kojima et al., 1997); Fast (Pottelette et al., 1999); and Cluster (Sigsbee et al., 2010). The observed Langmuir envelopes are highly structured and have occasionally the form of a chain of wave packets with nearly the same temporal scale. During such time intervals the power spectrum of the Langmuir wave form exhibits a double peak structure near $\omega_{\text {pe }}$, the electron plasma frequency.

The Langmuir envelope modulation has been clearly reproduced in a series of particle-in-cell (PIC) simulations of electron beam plasma interaction (Omura et al., 1996; Muschietti et al., 1996; Matsukiyo et al., 2004; Usui et al., 2005; Sauer and Sydora, 2012) and in electrostatic Vlasov simulations (Umeda, 2006; Silin et al., 2007; Daldorff et al., 2011), but the mechanism that causes the modulation and the nature of the wave packets has not yet been finally confirmed. Conventional nonlinear theory such as the modulation instability could be ruled out since the observed amplitude levels of the Langmuir waves did not exceed the relevant threshold. Parametric decay instability, which involves low-frequency ion acoustic waves in a three-wave interaction, has been considered as another candidate. Ion acoustic activity has been seen in PIC simulations as well as in observations, but the ion acoustic frequencies are too low to be responsible for the double peak in the power spectrum. As an alternative model, the so-called "nonlinear trapping theory" has been developed and applied to explain the modulation (Muschietti et al., 1996; Akimoto et al., 1996; Usui et al., 2005). This approach accounts for the trapping of beam electrons in the excited Langmuir wave field, which affects the growth and quenches the instability locally. This effect is suggested by the authors to be responsible for the Langmuir modulation, but conclusive support for this suggestion has not yet been given.

In this brief report we present a simple theoretical approach to the topic that is primarily based on linear theory. The simplification is suggested by PIC simulations and Vlasov-Poisson simulations, which provide evidence that the weak beam instability evolves in two main phases: a short initial interval in which the wave amplitude increases exponentially until saturation, and a longer period with significant variations in amplitude. Within these two phases the Langmuir waves are described in terms of their linear dispersion properties, where during the growth period only the exponential amplitude increase of unstable modes is considered. Nonlinear processes participate only phenomenologically in the model, as a mechanism that stops the growth and flattens 
the beam velocity distribution to a plateau. Intense linear and nonlinear investigations of the growth phase, in which the beam electron velocity distribution undergoes substantial changes, have been presented by Dum $(1989,1990 \mathrm{a}, \mathrm{b})$. This work covers several topics; among them are the variety of frequencies/bandwidths that can be excited by the instability depending on the parameters of beam and background electrons, the change of dispersive properties of the beamplasma system during the growth, and the transition between the fluid-like, reactive character of the instability for cold electrons and the classical kinetic bump-on-tail instability.

The evolution of the Langmuir wave field from thermal noise is treated by Fourier transform methods where the continuous Fourier integrals are approximated by finite sums (discrete Fourier transform). Then the fast Fourier transform (FFT) algorithm applies, which reduces the numerical expense. A set of plasma parameters is used that is suitable for qualitative comparison with PIC simulations and observations. The results of the model calculations suggest that typical features of the Langmuir wave modulation can be explained by linear theory.

In Sect. 2 we start with a recall of the fully kinetic dispersion properties of Langmuir waves during the growth period and after saturation. These results are the basis for the field calculation via FFT technique. A comparison with observations is presented in Sect. 3. Finally we consider in Sect. 4 the somewhat singular case in which the instability operates only very localized. The longitudinal field launched by a point-like instability may be considered as Green's function approach to the topic.

\section{Dispersion theory and model for field calculation}

When an electron beam propagates along the magnetic field in a magnetized plasma (or in a magnetic field-free plasma), electrostatic fields near the electron plasma frequency become unstable. Langmuir waves with wave numbers from an interval $k_{\min }<k<k_{\max }$ may be excited according to the predictions of the dispersion relation, with $k_{0}$, the value for the fastest growing mode, within this interval. Figure 1 displays solutions of the dispersion relation for a beam plasma system with Maxwellian velocity distributions of background and beam electrons.

$$
1-\frac{\omega_{\mathrm{pe}}^{2}}{2 k^{2} v_{\text {the }}^{2}} Z^{\prime}\left(\zeta_{\mathrm{c}}\right)-\frac{\omega_{\mathrm{pb}}^{2}}{2 k^{2} v_{\mathrm{thb}}^{2}} Z^{\prime}\left(\zeta_{\mathrm{b}}\right)=0
$$

with $\zeta_{\mathrm{e}}=\omega / \sqrt{2} k v_{\text {the }}, \zeta_{\mathrm{b}}=\left(\omega-k V_{\mathrm{b}}\right) / \sqrt{2} k v_{\text {thb }}$ and $Z$ the plasma dispersion function, for a typical set of parameters $\left(V_{\mathrm{b}} / v_{\text {the }}=7, n_{\mathrm{b}} /\left(n_{\mathrm{e}}+n_{\mathrm{b}}\right)=0.05, v_{\text {thb }} / v_{\text {the }}=1\right) . V_{\mathrm{b}}$ is the beam velocity and the subscripts "e" and "b" denote core and beam electrons. The natural normalizing quantities for frequency, wavenumber/distance and velocity are $\omega_{\text {pe }}$, the Debye length $\lambda_{\text {De }}=v_{\text {the }} / \omega_{\text {pe }}$, and $v_{\text {the }}$, respectively. Although
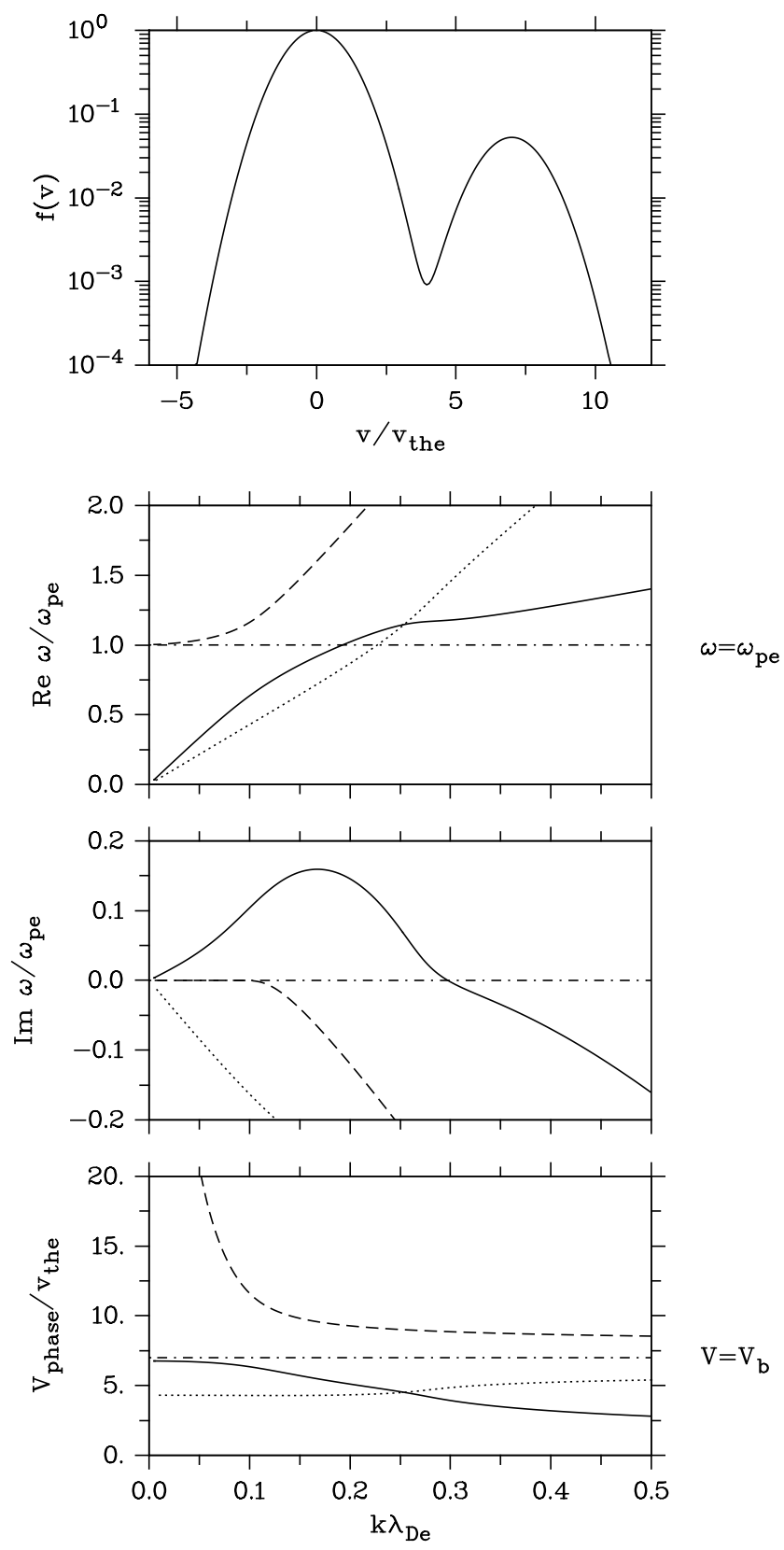

Fig. 1. Solutions of the dispersion relation for an electron beamplasma system with Maxwellian distribution functions for beam and background electrons (top panel). Solid line: unstable mode. Beam velocity $V_{\mathrm{b}} / v_{\text {the }}=7$, beam density $n_{\mathrm{b}} /\left(n_{\mathrm{e}}+n_{\mathrm{b}}\right)=0.05$, same temperature of beam and background electrons: $v_{\text {thb }} / v_{\text {the }}=1$; the maximum growth rate is seen to occur for $k \lambda \mathrm{De}_{\mathrm{De}} \approx 0.175$.

we performed runs with several other parameter combinations, we present here results mainly from the set above to demonstrate the capability of our model. Similar parameters have been used in PIC simulations. The wave growth is limited through saturation of the instability via nonlinear processes. At the end of the growth phase, the situation of 
the plasma is, roughly speaking, as follows: (1) an ensemble of Langmuir waves is present with wavenumbers from the unstable region $k_{\min }<k<k_{\max }$ and amplitudes according to the $k$-dependent growth rate; (2) the beam electrons have been scattered into a broader velocity range and their distribution function forms a plateau around the initial beam velocity, as it is known for the weak beam case $n_{\mathrm{b}} \ll n_{\mathrm{e}}$.

The Langmuir wave pattern within the instability region is influenced by random processes, because the waves grow from noise of the background plasma. As a consequence, even if all parameters controlling the instability are known, one cannot predict definite spatial or temporal profiles of the evolving Langmuir wave field. This principal uncertainty is incorporated in our analysis by introducing an initial low level field $E_{\mathrm{r}}(x)$, which varies randomly around zero with uniform amplitude. Its Fourier transform,

$E_{\mathrm{r}}(k)=\int_{-\infty}^{\infty} E_{\mathrm{r}}(x) \exp (-i k x) \mathrm{d} x$

is a function of the same type, i.e., also varying irregularly within an interval including zero. In order to check whether this choice of $E_{\mathrm{r}}(x)$ is consistent with general properties of longitudinal high-frequency fluctuations in a Maxwellian plasma, we recall the corresponding spectral power density (Bekefi, 1966, p. 109):

$P(k, \omega)=\frac{\kappa T_{\mathrm{e}}}{\pi \omega \varepsilon_{0}} \operatorname{Im} \frac{1}{K(k, \omega)}$

with $K(k, \omega)=1-1 /\left(2 \lambda_{\mathrm{De}}^{2}\right) Z^{\prime}\left(\zeta_{\mathrm{e}}\right)$ the longitudinal dielectric coefficient. $P(k, \omega)$ peaks at the solutions $\omega(k)$ of the dispersion relation $K(k, \omega)=0$. The power spectrum $P(k)$ of purely spatial fluctuations follows by integration of Eq. (3) over $\omega$, which can easily be carried out assuming small Landau damping. The result is $P(k)=\kappa T_{\mathrm{e}} / \epsilon_{0}$; i.e., the spatial power spectrum does not depend on $k$ in the wavelength region of interest. This result confirms the choice of $E_{\mathrm{r}}(x)$ to be reasonable. An orientation for the choice of the fluctuation intensity may be given by the thermal noise level in an Maxwellian plasma. For $T_{\mathrm{e}}=10 \mathrm{eV}$ and a density $n_{\mathrm{e}}=4 \mathrm{~cm}^{-3}$, one finds a level of the order of $10^{-3} \mathrm{mV} / \mathrm{m}$ (Bekefi, 1966, p. 125). This choice fixes the scaling of the field to $\mathrm{mV} / \mathrm{m}$. We treat the two main phases of the instability differently and adopt the following simplifications: during the growth period we ignore changes in the phase of the waves; i.e., we describe only the exponential amplitude increase of unstable modes. Propagation starts at the end of the growth without further amplification. During the growth period, $E_{\mathrm{r}}(k)$ is selectively amplified within the instability domain $k_{\min }<k<k_{\max }$ according to the growth rate $\gamma(k)$ predicted by the dispersion relation (Fig. 1). After saturation the new spectral function $E(k)$ may then be written as

$$
E(k)=\left(\begin{array}{cc}
E_{\mathrm{r}}(k) \exp \left[\gamma(k) t_{\mathrm{g}}\right] & \text { for } k_{\min }<k<k_{\max } \\
E_{\mathrm{r}}(k) & \text { elsewhere }
\end{array}\right.
$$

where $t_{\mathrm{g}}$ is the growth time. It is known from PIC simulations with similar parameters to be of the order of $50-100 \omega_{\mathrm{pe}}^{-1}$. We use here values $\omega_{\text {pe }} t_{\mathrm{g}}=40 \ldots 60$. We consider the spectral function $E(k)$ of Eq. (4) as initial condition for the spacetime evolution of the Langmuir field. The field $E(x, t)$ after saturation is then given by the inverse Fourier integral

$E(x, t)=\frac{1}{2 \pi} \int_{-\infty}^{\infty} E(k) \exp [-i \omega(k) t+i k x] \mathrm{d} k$

where $t$ counts the time after saturation. In this equation the knowledge of the dispersion law $\omega(k)$ after saturation is required. The situation shown in Fig. 1 is no longer applicable; instead, we need the dispersion properties of longitudinal waves in a Maxwellian electron distribution with a plateau around $V_{\mathrm{b}}$, as remnant of the beam. Figure 2 displays in the top panel such a distribution function in which the beam component of Fig. 1 is replaced by a plateau distribution of the same density, centered around the beam velocity. The plateau electrons are distributed according to the function

$$
\begin{aligned}
f_{p}(v)= & \frac{1}{A}\left[\operatorname{erf}\left(\frac{v+\Delta v-V_{\mathrm{b}}}{s}\right)\right. \\
& \left.-\operatorname{erf}\left(\frac{v-\Delta v-V_{\mathrm{b}}}{s}\right)\right] .
\end{aligned}
$$

It merges for $s \rightarrow 0$ into a water-bag distribution of extension $2 \Delta v$ in velocity space with center $V_{\mathrm{b}}$, and in a Maxwellian with $v_{\text {th }}=s / \sqrt{ } 2$ for $\Delta v \ll s$. The denominator $A$ normalizes $f_{p}(v)$ to one, i.e., $A=\int_{-\infty}^{\infty}[\operatorname{erf}((v+\Delta v) / s)-\operatorname{erf}((v-$ $\Delta v) / s)] \mathrm{d} v$. The dispersion relation associated with the combination of Maxwellian and plateau distribution (Eq. 6) can easily be derived. It reads

$$
1-\frac{\omega_{\mathrm{pe}}^{2}}{2 k^{2} v_{\text {the }}^{2}} Z^{\prime}\left(\zeta_{\mathrm{c}}\right)-\frac{\omega_{\mathrm{pb}}^{2}}{k^{2}} \frac{2}{A s}\left[Z\left(\zeta^{+}\right)-Z\left(\zeta^{-}\right)\right]=0
$$

with $\zeta^{+}=\left(\omega+k \Delta v-k V_{\mathrm{b}}\right) / s$ and $\zeta^{-}=\left(\omega-k \Delta v-k V_{\mathrm{b}}\right) / s$. The instability has disappeared, and besides the Langmuir oscillations for small $k$ another weakly damped Langmuir mode with frequencies near $\omega_{\text {pe }}$ exists in a wavenumber domain around $k_{0}$. This mode is supported by the plateau electrons, but it is almost identical to the standard Langmuir mode in the non-beam case. Its group velocity appears to be very close to the thermal velocity of the background electrons. A noticeable characteristic of the dispersion shown in Fig. 2 is the mode of acoustic type with $\omega \rightarrow 0$ for $k \rightarrow 0$. It can be placed into the category of electron acoustic waves. Its phase velocity is in the vicinity of the high-energy edge of the plateau distribution, and its damping is controlled by the slope of the distribution function in this region. In a simplified situation in which the plateau distribution is approximated by a shifted water-bag profile, the phase velocity coincides with the water-bag boundary velocity and the damping is almost zero. 

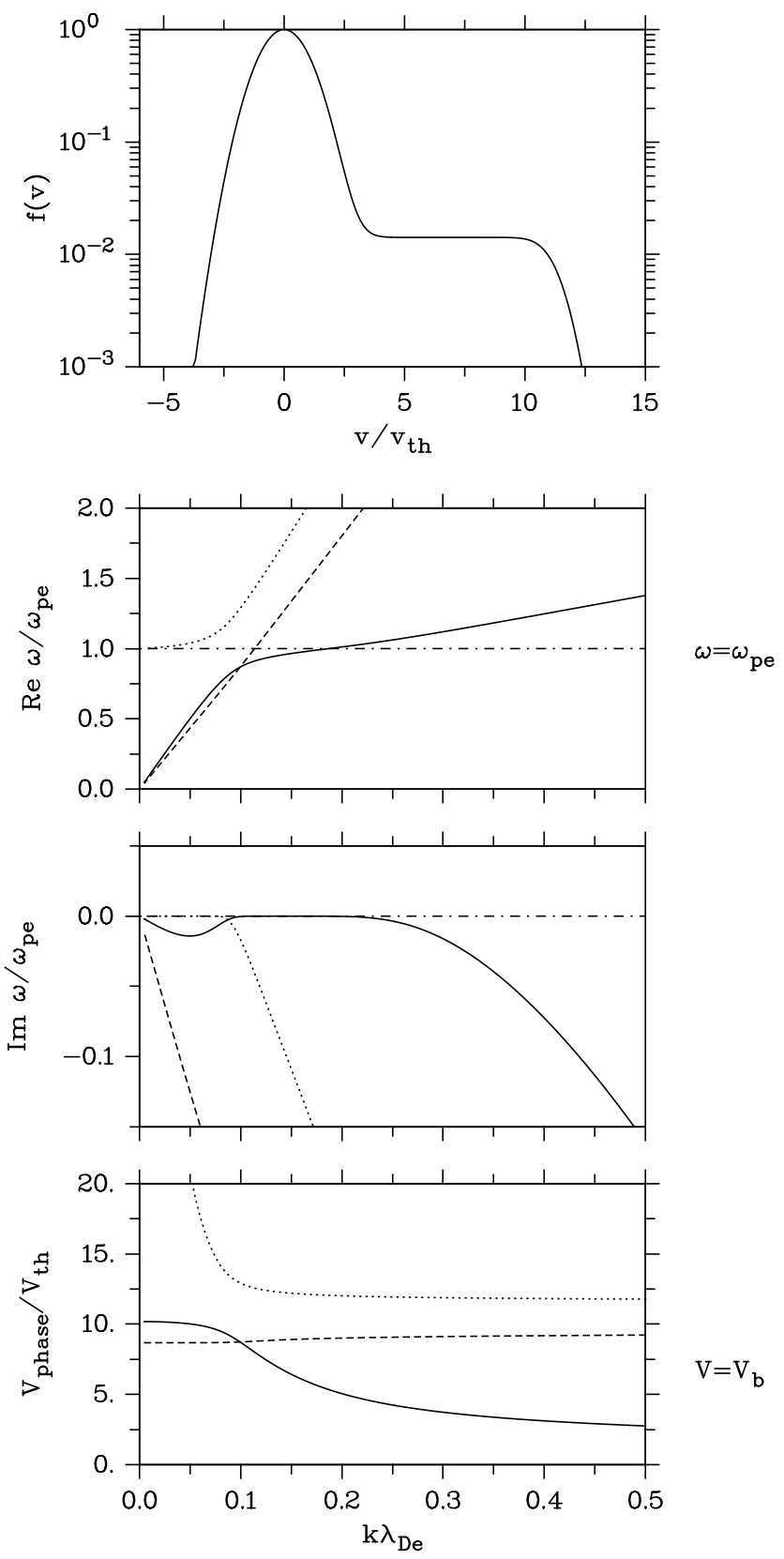

Fig. 2. Longitudinal wave dispersion for a Maxwellian electron velocity distribution with a plateau (top panel). Solid line: remnant of the unstable mode in Fig. 1. Almost undamped propagation of Langmuir waves is possible with $\omega \approx \omega_{\text {pe }}$ in a wavenumber region around $k_{0}$, the wavenumber of the fastest growing mode in Fig. 1. Parameters for the plateau are $\Delta v=4.7$ and $s=1$.

Results of the inverse Fourier transform Eq. (5) are shown in Figs. 3 and 4. Figure 3 displays the Langmuir wave form at the end of the growth period. Shown are spatial profiles of the real part of the field (top panel), the field envelope (middle) and the power spectrum (bottom). The numerical values of the growth rate $\gamma(k)$ have been extracted from Fig. 1 and those for the complex frequency $\omega(k)$ from Fig. 2 . The level of the initial fluctuation power spectrum is indicated in Fig. 3 for larger wavenumbers $k \lambda_{\text {De }}>0.3$. The quasi-regular spatial wave forms shown in Fig. 3 are similar to those seen in PIC simulations and Vlasov-Poisson simulations. Characteristic features are the significant amplitude modulation and the formation of Langmuir wave packets with a scale of 5-10 wavelengths of the mode with the largest temporal growth rate, and zero points of the envelope. The field amplitude reaches values in the $\mathrm{mV} / \mathrm{m}$ range, which is consistent with the order of magnitude seen in observations (Hospodarsky et al., 1994; Kellog et al., 1996; Kojima et al., 1997; Pottelette et al., 1999; Sigsbee et al., 2010). We emphasize that the strong modulation seen here comes about only by the beating of Langmuir waves with different wavenumbers and different amplitudes. Nonlinear processes are not involved in the formation of the highly structured Langmuir wave pattern. The power spectrum reflects roughly the $k$-variation of $\exp \left[\gamma(k) t_{\mathrm{g}}\right]$ with the growth rate shown in Fig. 1. Figure 4 displays changes in the spatial Langmuir wave form when time elapses. The whole structure moves in direction of the beam with the group velocity $\mathrm{d} \omega / \mathrm{d} k \approx v_{\text {the }}$, but during the motion the waveform undergoes deformations due to dispersion and, to a lesser extent, also by Landau damping.

The integral expression in Eq. (5) is suitable to examine also the temporal wave modulation. Figure 5 displays time profiles at the position $x / \lambda_{\mathrm{De}}=20$ up to $t=1000 \omega_{\mathrm{pe}}^{-1}$. Shown are the time variation of the real part of the field (top panel), the envelope (middle) and the power spectrum (bottom). The latter exhibits a weakly developed double peak structure near $\omega_{\text {pe }}$, which reflects the quasi-periodic amplitude modulation. In addition, Fig. 6 displays time profiles for another four positions demonstrating the wide variety of waveforms and the randomness of the amplitude modulation.

\section{Discussion, comparison with observations}

In this section we first discuss briefly to what extent the waveforms shown above are modified when parameters change. The beam velocity as dominant parameter determines the wavenumber $k_{0}$ of the most unstable mode whereby increasing $V_{\mathrm{b}}$ results in decreasing $k_{0}$ and vice versa. In the limit of cold electrons, there is the simple relation $k_{0} V_{\mathrm{b}}=\omega_{\text {pe }}$, which gives approximately $k_{0} \lambda_{\mathrm{De}} \approx$ $v_{\text {the }} / V_{\mathrm{b}}$ in the general case. In a run with higher beam velocity, $V_{\mathrm{b}} / v_{\text {the }}=15$, the wavenumber $k_{0}$ is reduced accordingly and the wave packets expand in size. This implies an increase of the time duration of the packet in temporal representation as well, because the propagation velocity $\sim v_{\text {the }}$ of the structure remains the same. Besides the decrease in $k_{0}$, a higher beam velocity produces higher growth rates and supports the instability. On the other hand, a lower beam velocity shifts the wavenumber of the fastest growing mode to higher values, thus creating smaller wave packet sizes and shorter event 

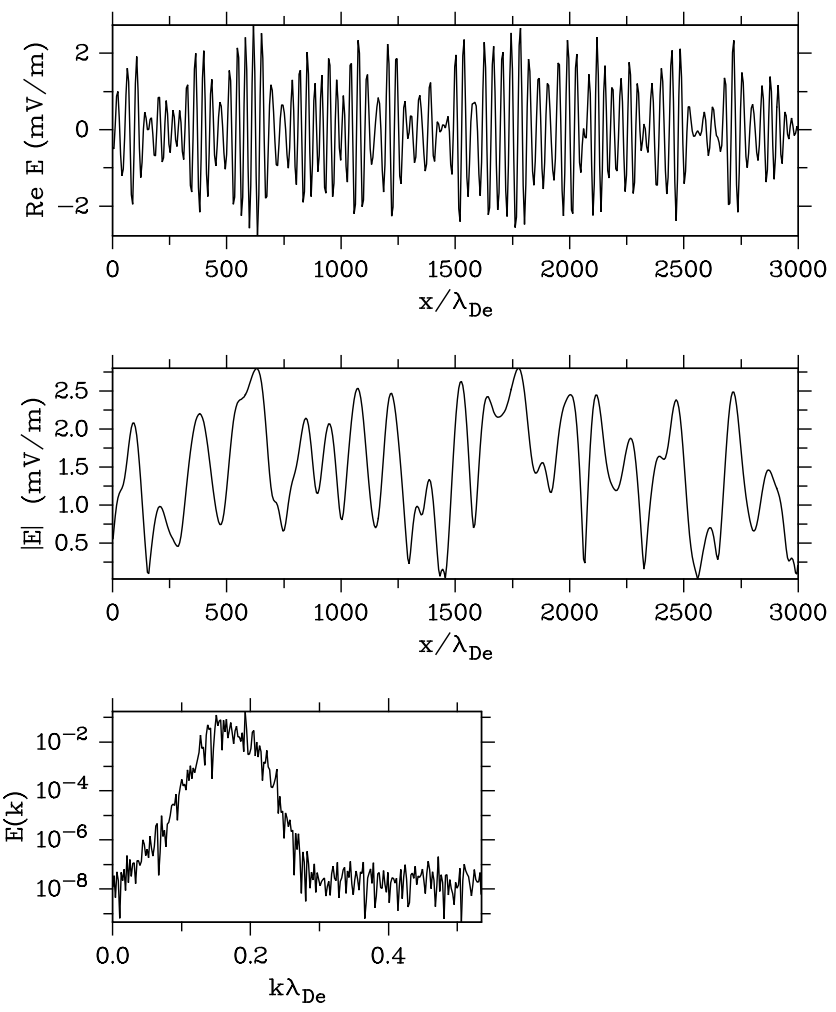

Fig. 3. Spatial representation of amplitude-modulated Langmuir waves grown out from noise, evaluated from the inverse Fourier integral Eq. (5) for $t=0$, i.e., at the end of the growth period. Real part of $E$ (top panel), envelope $|E|$ (middle), and power spectrum $E(k)$ (bottom). The power spectrum reproduces the spectral function of Eq. (4) and indicates the level of the noise power spectrum for $k \lambda \mathrm{De}>0.3$.

durations. In addition, Landau damping, which becomes active for $k \lambda_{\text {De }} \geq 0.3$, may affect the short wavelength part of the excited Langmuir wave spectrum and may reduce the field amplitude. In a run with $V_{\mathrm{b}} / v_{\text {the }}=4$, the wavenumber for maximum growth is shifted to $k_{0} \lambda_{\mathrm{De}} \approx 0.3$. In this case amplification and damping nearly compensate each other and no waveform like that in Fig. 3 develops.

Both beam temperature and beam density affect the growth rates, i.e., the peak electric field strength, rather than the wavenumber of maximum growth. Spatial and temporal scales of the amplitude modulation are less sensitive to changes in $v_{\text {thb }}$ and $n_{\mathrm{b}}$. In particular, beat-type waveforms develop even for very thin beams.

As far as comparison with measurements is concerned, it is restricted to time profiles because only one-point observations are available. As expected for Langmuir waves, the power spectrum in both observed and calculated time records peaks always near $\omega_{\text {pe }}$. Therefore, the main interest is rather focused on the time scale of the amplitude modulation and wave packets. Table 1 contains durations $\Delta t$ of wave packets observed by several spacecraft. They are in the range of

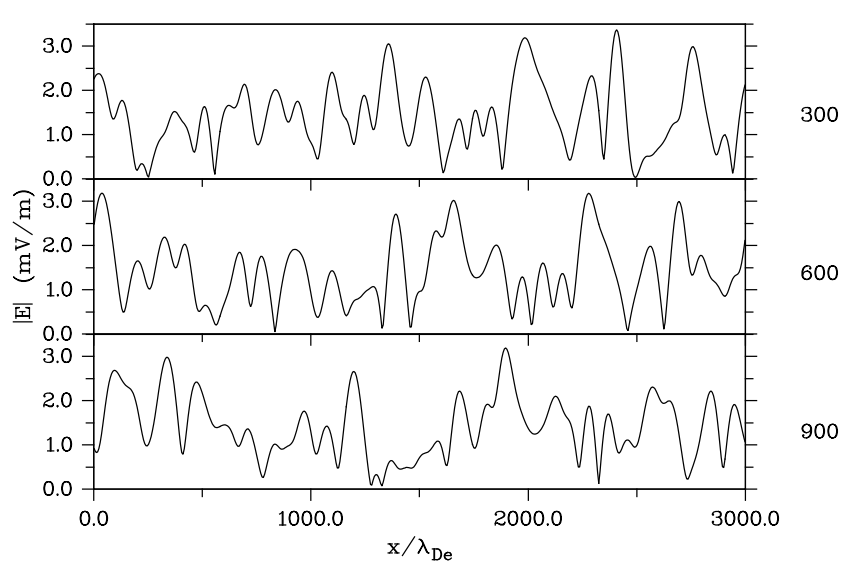

Fig. 4. Spatial profile of the field envelope for another three times, illustrating the modulation during propagation.

some hundreds of Langmuir cycles. The model calculations produce typical time scales $\Delta t=100-200 \omega_{\mathrm{pe}}^{-1}$ for the standard parameter set, as seen in Figs. 5, 6. They expand to $\Delta t=200-400 \omega_{\mathrm{pe}}^{-1}$ for a faster beam with $V_{\mathrm{b}} / v_{\text {the }}=15$ (not shown). Thus the time scales predicted by the model are consistent with those seen in observations.

We may conclude that the simple Fourier analysis of the weak beam instability clearly reproduces spatial and temporal Langmuir amplitude modulation and the formation of wave packets of variable length. The linear model fails, however, in an important point: it does not produce regular chains of wave packets with the same time scale during the evolution. Such waveforms are seen in observations, with a double peak near $\omega_{\text {pe }}$ as signature in the corresponding power spectra. Figure 5 displays such a power spectrum, but the double peak is not well enough developed compared with that, e.g., seen in Fig. 3d of Sigsbee et al. (2010).

The occurrence of relatively coherent strings of wave packets is suggestive of a nonlinear process. One might think that the spatial gradients in the field envelope (displayed in Fig. 3), which give rise to ponderomotive forces, may initiate nonlinear processes of modulation type as described by the nonlinear Schrödinger equation

$i \frac{\partial E}{\partial t}+\frac{3}{2} \frac{v_{\text {the }}^{2}}{\omega_{\mathrm{pe}}} \frac{\partial^{2} E}{\partial x^{2}}+\frac{1}{8} \epsilon_{0} \omega_{\mathrm{pe}} E \frac{|E|^{2}}{n_{0} \kappa\left(T_{\mathrm{e}}+T_{\mathrm{i}}\right)}=0$

(see, for example, Pecseli, 1985). Here the real electric field $\hat{E}$ is separated in a rapid and a slow time scale, according to $\hat{E}=1 / 2\left[E(x, t) e^{-i \omega_{\mathrm{pe}} t}+\right.$ c.c. $]$ where $E(x, t)$ accounts for both the slow time variation and the full spatial variation. The nonlinear term, however, is for the relevant parameters (field strength, density, electron temperature) more than ten orders of magnitude smaller than the linear terms, thus unable to affect the evolution. Applied to the initial field profile of Fig. 3, the linear terms of Eq. (8) reproduce the time 
Table 1. Duration $\Delta t$ of Langmuir wave packet events.

\begin{tabular}{lll}
\hline & their Fig. & $\Delta t\left(\omega_{\mathrm{pe}}^{-1}\right)$ \\
\hline $\begin{array}{l}\text { Gurnett et al. (1993) } \\
\text { Galileo }\end{array}$ & Fig. 4 & 390,550 \\
$\begin{array}{l}\text { Hospodarsky et al. (1994) } \\
\text { Galileo Venus }\end{array}$ & Fig. 4 & 445 \\
$\begin{array}{l}\text { Kellog et al. (1996) } \\
\text { Wind }\end{array}$ & Fig. 1 & 210 \\
$\begin{array}{l}\text { Kojima et al. (1997) } \\
\text { Geotail }\end{array}$ & Fig. 1b & 210 \\
$\begin{array}{l}\text { Pottelette et al. (1999) } \\
\text { Fast }\end{array}$ & Fig. 2 & 390 \\
$\begin{array}{l}\text { Sigbee et al. (2010) } \\
\text { Cluster }\end{array}$ & Fig. 3a, b, c & $250,315,950$ \\
\hline
\end{tabular}
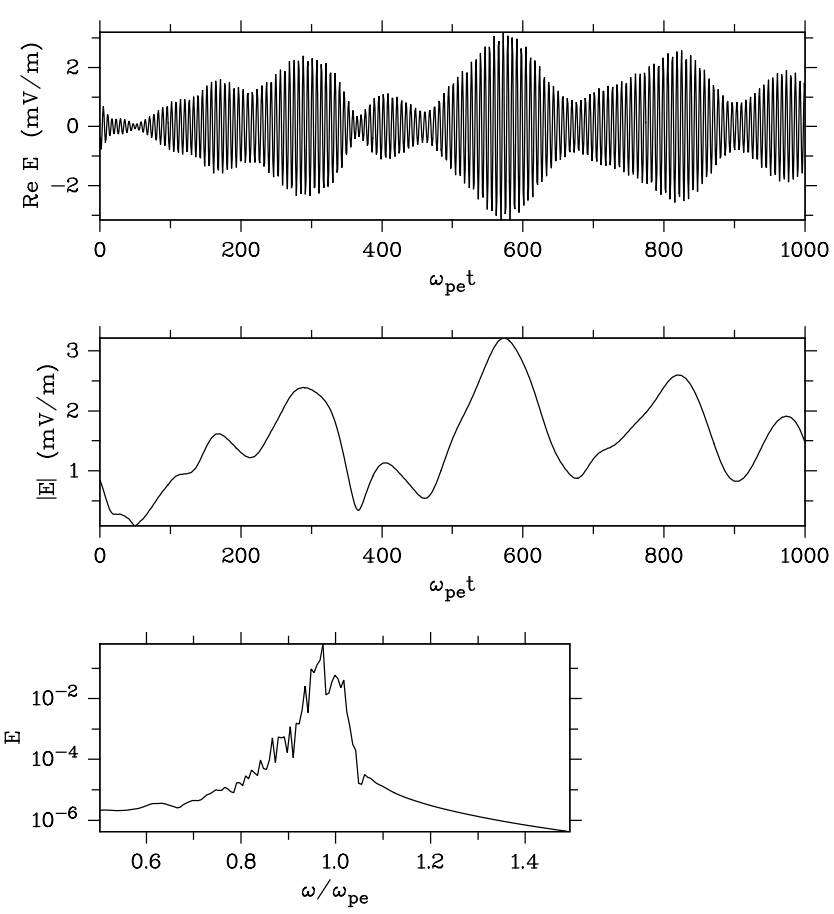

Fig. 5. Temporal representation of the amplitude-modulated Langmuir field at position $x / \lambda \mathrm{De}=20$ up to $\omega_{\mathrm{pe}} t=1000$. Real part (top panel), envelope (middle) and power spectrum (bottom). Note the (weakly developed) double peak near $\omega / \omega_{\text {pe }}=1$.

evolution displayed in Fig. 4 almost accurately. The source for the formation of more coherent strings of wave packets remains unclear.

It should finally be mentioned that the plateau in the electron velocity distribution has implications also for the lowfrequency region near to the ion plasma frequency. The electron acoustic mode continues to exist down to frequencies less than $\omega_{\text {pi }}$ with constant phase velocity and constant damping decrement $\operatorname{Im} \omega / \operatorname{Re} \omega$. This has been verified by solving the dispersion relation (1) including the ion term. Phase ve-

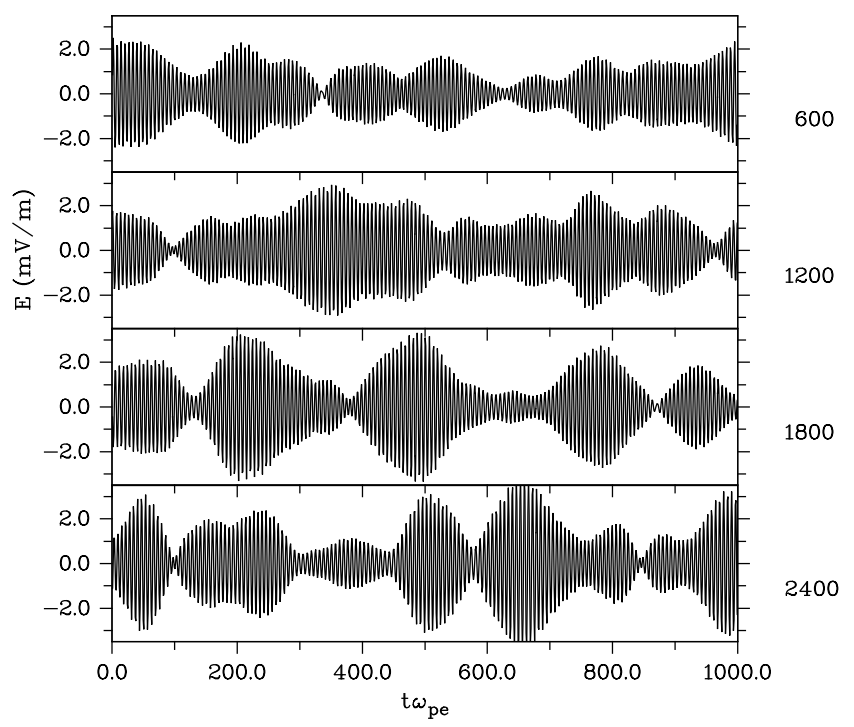

Fig. 6. Time variation of Re $E$ at another four positions.

locity and damping depend on the shape of the plateau distribution near to the high velocity boundary. In our case the mode appears as weakly damped ( $\operatorname{Im} \omega / \operatorname{Re} \omega \approx 0.04)$. This low-frequency electron acoustic mode may be a potential partner for nonlinear interaction with the excited Langmuir waves.

\section{Point-like instability}

In this section we briefly consider a somewhat academic special case of our model. The motivation is to eliminate the randomness inherent in the evolution of the instability in an extended region. To this end we let the width of the instability area shrink to zero and calculate the Langmuir wave field launched by a point-like source. With a field $E_{\mathrm{r}}(x)=E_{0} \delta(x)$ the integral Eq. (5) reduces to $E_{\mathrm{r}}(k)=E_{0}=$ const and the space-time evolution is given by

$E(x, t)=\frac{E_{0}}{2 \pi} \int_{-\infty}^{\infty} \exp \left[\gamma(k) t_{\mathrm{g}}-i \omega(k) t+i k x\right] \mathrm{d} k$

Figure 7 displays the situation at $t=0$. Shown are the amplitude function $A(k)=\exp \left[\gamma(k) t_{\mathrm{g}}\right]$ in the integral (top panel), real and imaginary part of the field and the envelope. We find a Langmuir wave packet centered around the source consisting of a few spatial cycles with a wavelength given by $k_{0}$, the wave number for the fastest growing mode. Since the shape of $A(k)$ is approximately Gaussian, the same is true for the envelope of the Fourier transform, and we may write

$E(x, 0) \approx|E(x, 0)| \exp [-i k x]$ 

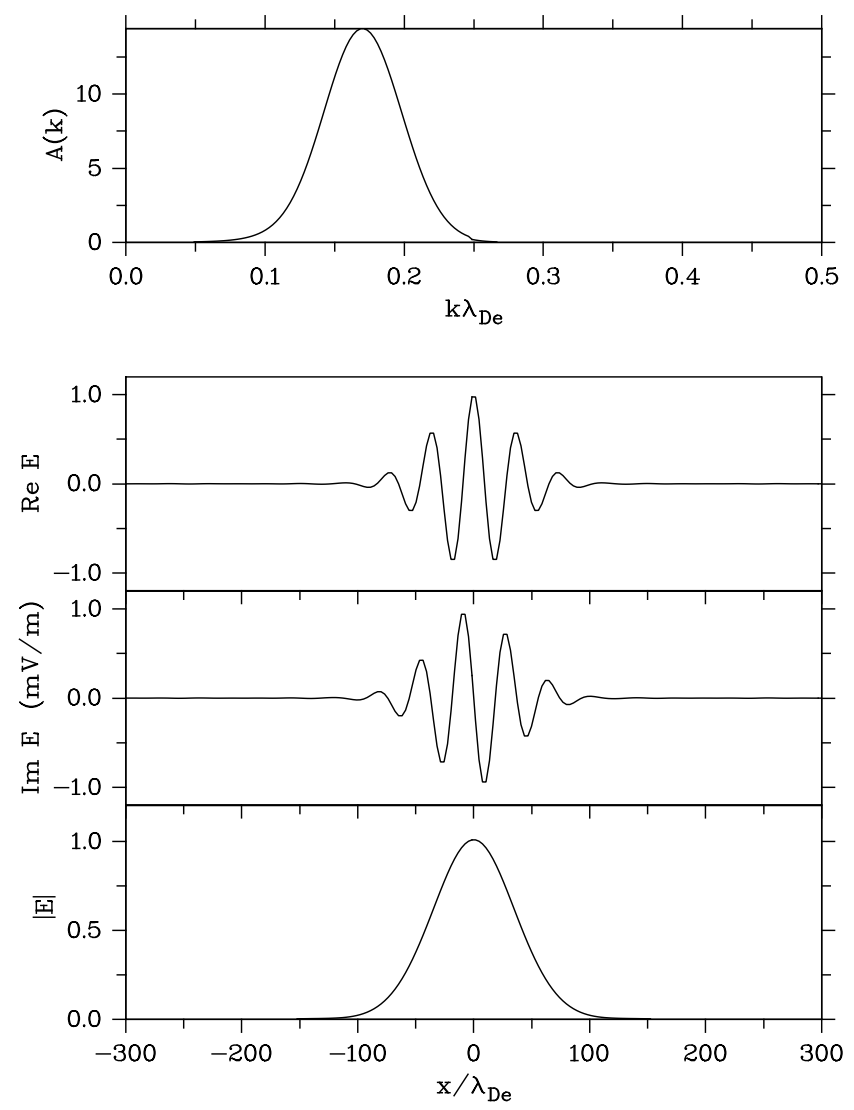

Fig. 7. Isolated wave packet launched by a point-like instability: amplitude $A(k)=\exp \left[\gamma(k) t_{\mathrm{g}}\right]$ of the Fourier transform (top panel); spatial field profiles $\operatorname{Re} E, \operatorname{Im} E$ (middle) and field envelope (bottom), $t_{\mathrm{g}}=50 \omega_{\mathrm{pe}}^{-1}$, other parameters the same as in Fig. 1.

according to the shifting rule of Fourier transform $H(k-$ $\left.k_{0}\right) \Longleftrightarrow h(x) \exp \left[-i k_{0} x\right]$ where $h$ and $H$ are transform pairs. The spatial scale of the packet is controlled by the width of the instability domain in $k$-space. A narrow growth range produces a broad wave pulse and vice versa. The isolated wave packet propagates with the group velocity at $v_{\mathrm{gr}} \approx v_{\text {the }}$ and relaxes due to dispersion and Landau damping. The isolated wave pulse is suggested to play the role of Green's function for the evolution of the instability in an extended region.

\section{Summary}

Langmuir waveforms observed in space plasmas show a considerable variety. Most of them exhibit an irregular, random structure, consisting of wave periods of variable length. Occasionally, however, the waveform is almost coherently modulated and has the form of a string of wave packets with nearly the same time scale. In this report Langmuir wave modulation is examined by a linear model that is based on the dispersion properties of the waves involved in the beamplasma interaction. This strategy is suggested by PIC simu- lations and Vlasov-Poisson simulations, which provide evidence that the gentle beam instability evolves in two main phases. In an initial interval the Langmuir wave field grows exponentially until saturation and the beam velocity distribution relaxes to a plateau. In a subsequent longer period, no further amplification takes place and the wave amplitude exhibits significant variations. In our linear model we explain the amplitude modulation on the basis of dispersion properties of the Langmuir waves involved in the instability and suggest that the modulation is due to the beating of waves with different wave numbers and amplitudes that have grown from noise during the initial period of the instability. Nonlinear processes are only phenomenologically involved, as mechanisms for saturation and flattening the beam velocity distribution. We have used a representative parameter set to demonstrate the capability of the model. The fully kinetic beam plasma dispersion relation is solved to get the growth rates of the broad band of unstable waves and their amplitudes at the end of the growth period. The further spacetime evolution of this wave ensemble is calculated by FFT technique using the solution of the dispersion relation for the plateau distribution. The model yields amplitude modulation both in space and time quite similar to that seen in PIC/Vlasov simulations or in observations. The range of time scales of wave packets is consistent with that of one-point observations of several spacecraft. It has become evident that the modulation is primarily a linear phenomenon, thus can be seen with weak electric fields. We can conclude that the linear dispersion model accounts for basic features of the Langmuir modulation, but fails in reproducing coherent structures in which wave packets repeat their shape periodically. To analyze such waveforms it seems necessary to invoke nonlinear processes.

Acknowledgements. The author would like to express his thanks to K. Sauer for pointing out the problem and for valuable discussions during the preparation of the manuscript.

After submitting the manuscript a way was found to remove a crucial limitation of the model, namely to describe only the irregular amplitude modulation. The linear approach can be extended to explain the coherent modulation as well. This will be the subject of a follow-up paper.

Topical Editor I. Daglis thanks S. C. Buchert and one anonymous referee for their help in evaluating this paper.

\section{References}

Akimoto, K., Omura, Y., and Matsumoto, H.: Rapid generation of Langmuir wave packets during electron beam-plasma instabilities, Phys. Plasmas, 3, 2559-2563, 1996.

Bekefi, G.: Radiation Processes in Plasmas, John Wiley \& Sons, Inc., New York, London, Sydney, 1966.

Daldorff, L. K. S., Pecseli, H. L., Trulsen, J. K., Ulriksen, M. I., Eliasson, B., and Stenflo, L.: Nonlinear beam generated plasma 
waves as a source for enhanced plasma and ion acoustic lines, Phys. Plasmas, 18, 052107, doi:10.1063/1.3582084, 2011.

Dum, C. T.: Transition in the Dispersive Properties of Beam-Plasma and Two-Stream Instabilities, J. Geophys. Res., 94, 2429-2442, 1989.

Dum, C. T.: Simulation Studies of Plasma Waves in the electron foreshock: The generation of Langmuir waves by a Gentle Bump-on-Tail Electron Distribution, J. Geophys. Res., 95, 80958110, 1990a.

Dum, C. T.: Simulation Studies of Plasma Waves in the electron foreshock: The Transition From Reactive to Kinetic Instability, J. Geophys. Res., 95, 8111-8122, 1990b.

Gurnett, D. A., Maggs, J. E., Gallagher, D. L., Kuhrt, W. S., and Scarf, F. L.: Parametric interaction and spatial collapse of beamdriven Langmuir waves in the solar wind, J. Geophys. Res. 86, 8833-8841, 1981.

Gurnett, D. A., Hospodarsky, G. B., Kurth, W. S., Williams, D. J., and Bolton, S. J.: Fine structure of Langmuir waves produced by a solar electron event, J. Geophys. Res., 98, 5631-5637, 1993.

Hospodarsky, G. R., Gurnett, D. A., Kurth, S. W., Kivelson, M. G., Strangeway, R. J., and Bolton, S. J.: Fine structure of Langmuir waves observed upstream of the bow shock at Venus, J. Geophys. Res., 99, 13363-13371, 1994.

Kellog, P. I., Morison, S. J., Goetz, K., Howard, R. L., Bougeret, J. L., and Kaiser, M. L.: Early wind observations of bow shock and foreshock waves, Geophys. Res. Lett., 23, 1243-1246, 1996.

Kojima, H., Furuya, H., Usui, H., and Matsumoto, H.: Modulated electron plasma waves observed in the tail lobe: Geotail waveform observations, Geophys. Res. Lett., 24, 3049-3052, 1997.

Matsukiyo, S., Treumann, R. A., and Scholer, M.: Coherent waveforms in the auroral upward current region, J. Geophys. Res., 109, A06212, doi:10.1029/2004JA010477, 2004.

Muschietti, L., Roth, I., and Ergun, R. E.: On the formation of wave packets in planetary foreshocks, J. Geophys. Res., 101, 1560515613, 1996.
Nariyuki, Y. and Umeda, T.: On the nonlinearity of the Langmuir urbulence excitd by a weak electron beam-plasma interaction, Phys. Plasmas, 17, 054506, doi:10.1063/1.3425872, 2010.

Omura, Y., Matsumoto, H., Miyake, T., and Kojima, H.: Electron beam instabilities as generation mechanism of electrostatic solitary waves in the magnetotail, J. Geophys. Res., 101, 2685-2697, 1996.

Pecseli, H.: Solitons and weakly nonlinear waves in plasmas, IEEE T. Plasma Sci., Vol. PS-13, No. 2, 1985.

Pottelette, R., Ergun, R. E., Treumann, R. A., Berthomier, M., Carlson, C. W., McFadden, J. P., and Roth, I.: Modulated electronacoustic waves in auroral density cavities: Fast observations, Geophys. Res. Lett., 26, 2629-2632, 1999.

Sauer, K. and Sydora, R.: Mode crossing effects at electron beamplasma interaction and related phenomena, Plasma Phys. Control. Fusion, 54, 124054, doi:10.1088/0741-3335/54/12/124045, 2012.

Sigsbee, K., Kletzing, C. A., Pickett, J. S., Gurnett, D. A., Schwartz, S. J., Levebvre, B., Lucek, E., Fazakerley, A. N., and Kucharek, H.: Characteristics of Langmuir electric field waveforms and power spectra exhibiting nonlinear behavior in Earth's foreshock, J. Geophys. Res., 115, A10251, doi:10.1029/2009JA014948, 2010.

Silin, I., Sydora, R., and Sauer, K.: Electron beam-plasma interaction: Linear theory and Vlasov-Poisson simulations, Phys. Plasmas, 14, 012106, doi:10.1063/1.2430518, 2007.

Umeda, T: Vlasov simulation of amplitude modulated Langmuir waves, Phys. Plasmas, 13, 092304, doi:10.1063/1.2348088, 2006.

Usui, H., Furuya, H., Kojima, H., Matsumoto, H., and Omura, Y.: Computer experiments ofamplitude-modulated Langmuir waves: Application to Geotail observation, J. Geophys. Res., 110, A06203, doi:10.1029/2004JA010703, 2005. 